\title{
FORMAP : A SIMPLE AND FAST PHOTOGRAMMETRY FRAMEWORK FOR DIRECT GEO-REFERENCING SYSTEMS
}

\author{
G. Truong Nguyen ${ }^{1, *}$, N. Seube ${ }^{2}$ \\ ${ }^{1}$ Geown France, 13 Avenue de l'Europe, 31520 Ramonville St-Agne, FRANCE, giang.nguyen@geown.io \\ ${ }^{2} 3051$ rue du Plateau, J7V 8P2 Vaudreuil-Dorion, Québec, CANADA - nicolas.seube@ geown.io
}

\section{Commission II}

KEY WORDS: Photogrammetry, Direct georeferencing, Orthophoto, UAV mapping

\begin{abstract}
:
This paper presents FORMap (Fast Ortho Mapping) a simple, automatic, fast and accurate commercial photogrammetry processing software for Unmanned Aerial Vehicles (UAV) imagery equiped with Direct Georeferencing (DG) technology. DG technique allows user to directly geo-reference the acquisition without the use of Ground Control Points (GCP) by providing image external orientation (EO) parameters in a mapping frame. However, it requires a sensor of relatively high quality to provide an accurate EO with each image shot, which is somehow limited by the light weight of UAV payloads. FORMap makes use of EO information delivered by DG as an a priori information to accelerate its photogrammetric processing. We present the functionalities and some application of FORMap in the field of UAV mapping. We evaluate its accuracy and its robustness on several datasets. Test result shows that FORMap is robust for 3D scene reconstruction despite of inaccuracies of DG input data. It is also faster than standard digital photogrammetry solution based on SfM (Structure from Motion) approach and can provide orthophotos and dense point cloud in quasi real-time.
\end{abstract}

\section{INTRODUCTION}

\subsection{Direct geo-referencing in photogrammetry}

Low-cost UAV (Unmanned Aerial Vehicles) mapping systems can provide aerial images with external orientation (EO) parameters thanks to their integrated GNSS (Global Navigation Satellite System) and IMU (Inertial Measurements Unit) sensor. These systems - called direct geo-referencing (DG) — have a major advantage in determining the coordinates of ground points by direct triangulation of bundles. Using DG information, we can geo-reference the scene without the use of GCPs (Ground Control Point). However, inaccurate EO parameters given by small UAV platform could be a problem if we use them directly for 3D scene restitution. Aerial triangulation (AT) can then be used to enhance the precision of EO determination, especially in the case of non-post processed of navigation data. The combination between AT, DG and optimized strategies in tie-points extraction, dense matching and orthophoto rectification significantly accelerates the photogrammetric processing time.

The development of UAV (Unmanned Aerial Vehicles) technology in the recent years brings us a large choice of drones with DG technology (Eisenbeiß, 2009), from the professional grade such as Microdrones systems (Mian et al., 2015, Pérez et al., 2013, Sauerbier et al., 2011) to a popular low-cost solution such as DJI Phantom platform (Taddia et al., 2019, Peppa et al., 2019). However, the accuracy of geo-referencing depends on the quality of integrated sensors, on the time synchronization and finally on boresight and lever arm calibration. (Gabrlik et al., 2016).

DG data can be embedded into image metadata (by geotagging). Exchangeable Image File Format (EXIF) (Association

\footnotetext{
${ }^{*}$ Corresponding author
}

et al., 2010) standard is widely used for this purpose. It is extended by Adobe with Extensible Metadata Platform (XMP) standard to provide more flexibility to add user-defined tags (Ball, Darlington, 2007, Specification, 2005) (e.g. embedding image's attitude, gimbal orientation).

Most of commercial digital photogrammetry solutions such as Pix4D or Agisoft automatically extract these data from image metadata for their processing needs. Software can get image position (latitude, longitude, altitude) and attitude (roll, pitch, yaw) and use of them as an absolute EO parameters for georeferencing purposes or to optimize the image network geometry.

Post processing of DG navigation data can enhance the precision of absolute geo-referencing (Rabah et al., 2018). With a rigorous lever-arm calibration between GNSS and the camera optical center, absolute geo-referencing could reach a centimetric precision (Daakir, 2017), even in some difficult overlapping configurations such as the one found in corridor mapping applications (Zhou et al., 2018).

\subsection{Aim of the paper}

This paper aims at introducing FORMap ${ }^{\circledR}$ : a commercial photogrammetry solution designed for UAV acquisition with DG technology. FORMap is developed by Geown, and focus on the simplicity of use and high processing speed. From a direct geo-referencing image dataset, without any user interaction, FORMap computes 3D dense point cloud and geo-referenced orthophoto. FORMap accelerate tie-points extraction process and bundle block adjustment by exploiting a priori EO data. Dense point cloud computation is accelerated based on initial depth estimated from a sparse tie-point surface model. Test results show that FORMap response time is compatible with quasi real-time applications of UAVs. 


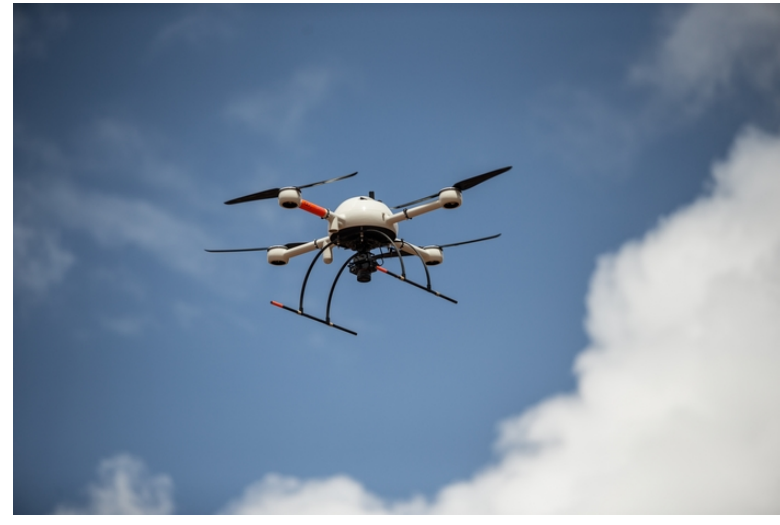

Figure 1. A Microdrones md4-1000DG system with Sony RX1-RII camera and APX-15 direct geo-referencing sensor.

UAV photogrammetry applications such as first response in disaster scenario, validity of data usability, quick preview on the field or a sample large scale survey require a fast map/3D models with less user interaction, at the price of degrading slightly the absolute direct georeferencing accuracy and quality of final result. FORMap is designed for these purposes.

The next section presents the photogrammetry data processing done within FORMap. The last section describes some test flight mission from several professional UAV manufacturers, from the data acquisition protocol to the processing workflow with FORMap, and the comparison of accuracy and processing time with Pix4D.

\section{DATA PROCESSING}

\subsection{Input and output data}

The FORMap software is available in Linux and Windows distributions, and cloud computing web-based service.

The software requires as input :

- Images with initial geolocation and orientation (EO)

- Initial camera calibration parameters (IO)

EO parameters can be imported into FORMap by following methods :

\section{- EXIF and XMP tags}

- Conventional text or .csv file

Image positions in latitude, longitude and elevation can be read from the corresponding image EXIF tag. Image orientation in degree given by roll, pitch, heading tags will be parsed from image XMP. In this case, FORMap automatically delivers results in a UTM mapping frame. The user can provide image positions and orientations in others mapping frame by a text or .csv file input. In all cases, the synchronization between images and EO parameters, the boresight and level-arm between sensors is defined by the user. Input EO and IO parameters can be entered in FORMap as approximate values.

The software delivers in output :
- Refined image EO parameters,

- Refined camera IO parameters,

- 3D dense point cloud,

- Geo-referenced orthophoto

without the need of GCPs.

\subsection{Processing steps}

FORMap does not employ a SfM (Structure from Motion) approach, which computes image EO and IO in a common 3D coordinate frame, by an incremental reconstruction process starts from an initial image pairwise or triplet (Bianco et al., 2018, Rupnik et al., 2017), or by a hierarchical approach (Toldo et al., 2015). FORMap achieves a robust BBA (Bundle Block Adjustment) by using initial EO parameters (given by DG) and IO parameters (givens by user, or taken from its camera calibration database).

General processing steps of FORMap are as follow:

1. Tie-points extraction

2. Bundle block adjustment

3. Dense matching

4. Orthophoto rectification

\section{EXPERIMENTS AND RESULTS}

\subsection{The evaluation procedure}

This section describes experiments and results obtained by FORMap on several UAV datasets. The evaluation is carried out on four datasets with different scene geometry, acquisition protocol and sensor type. The ground truth is available in one dataset, given by several GCPs measured by GNSS in RTK (Real Time Kinematic) mode. The main characteristics of these datasets are given in Table 1 and 2.

The UX11 and sensFly datasets were processed with two photogrammetry solutions: Pix4D and FORMap. As FORMap is designed to deliver a photogrammetric result as fast as possible in slightly degrading the accuracy, Pix4D has been set in Rapid mode to make a fair comparison. Image re-sampling factor for tie-point extraction, dense matching computation and GSD (ground sampling distance) of orthophoto are kept at the same values within the two softwares.

We provide the same input in the two software : geotagged images, camera IO parameters and camera distortion model (here using a five parameters model with 3 factors for radial distortion and 2 for tangential component).

Accuracy assessment is measured by euclidean distance between GCPs resulting from image triangulation and their true values. The two software use only the extracted tie-points and GNSS on image center constraints in BBA process. GCPs are only used for accuracy assessment.

To assure the same GCPs marks are used by the two software, we use the same GCPs marker file provided by the Pix4D 
marker tool ${ }^{1}$. The processing time will also be compared in the UX11 dataset.

The other datasets are only processed by FORMap to demonstrate the robustness of our software for various photogrammetry survey data type.

\begin{tabular}{|l|l|l|}
\hline Dataset & Highway & UX11 \\
\hline Geometry & UAV Corridor & UAV Grid \\
\hline Scene size [m]) & $1742 \times 2230$ & $1508 \times 1206$ \\
\hline Camera - UAV & md4-1000 & Delair UXI1 \\
& Sony RX1 RII 42Mpx & UX11-3B 20Mpx \\
\hline Focal length & $35 \mathrm{~mm}$ & x mm \\
\hline GCPs & No & $\begin{array}{l}13 \mathrm{GCP} \\
\text { RTK measure }\end{array}$ \\
\hline
\end{tabular}

Table 1. Dataset description - The highway and the UX11

\begin{tabular}{|l|l|l|}
\hline Dataset & senseFly & Inieres \\
\hline Geometry & UAV Grid & UAV Corridor \\
\hline Scene size $[\mathrm{m}]$ & $1508 \times 1206$ & $4058 \times 1857$ \\
\hline Camera - UAV & eBee X & Delair UX11 \\
\hline Focal length & Aeria X 24Mpx & UX11-3B 5Mpx \\
\hline GCPs & $14 \mathrm{GCP}$ & x mm \\
\hline
\end{tabular}

Table 2. Dataset description - The sensFly and the Inieres

\subsection{The UX11 and sensFly dataset}

The two datasets UX11 and senseFly cover the same area. It is a $1.8 \mathrm{~km}^{2}$ area with 13 GCPs distributed over the scene. GCPs are measured by GNSS RTK, with an uncertainty of $1 \mathrm{~cm}$ in the horizontal plane and $2 \mathrm{~cm}$ in elevation. Images GNSS positions were post-processed in PPK mode for these two datasets.

The UX11 dataset contains 2159 images from a 20 Mpx camera acquired with a Delair-Tech UX11 fixed-wing UAV. Images are taken by the UAV's integrated camera (sensor size 8.446 $\times 7.066 \mathrm{~mm}$ ). The sensFly dataset contains 676 images of 24 Mpx acquired with a eBeeX fixed wing UAV. The camera used is a senseFly Aeria X (sensor size ASP-C).

A snapshot of the dataset and the distribution of GCPs are shown in Figure 2. Details about the scene geometry and camera are given; in Table 1 and 2

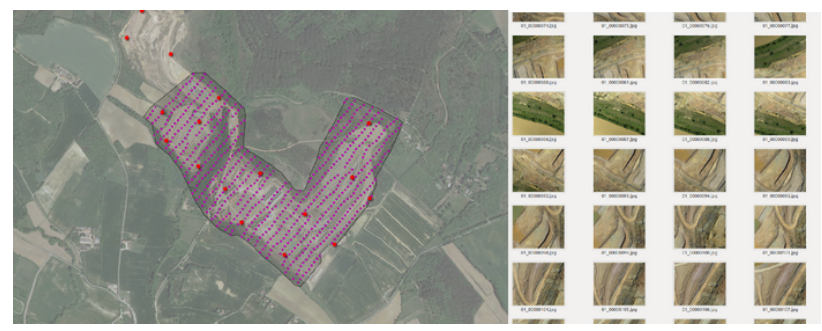

Figure 2. Left : the fight path (purple dots) and GCPs distribution (red dots) of UX11 and senseFly dataset. Right : some images of the scene extracted from the UX11 dataset.

Figure 3 shows a 3D dense point cloud of the scene reconstructed by Pix4D and FORMap on the UX11 dataset. All parts of the scene are reconstructed and the 2159 images are calibrated successfully by both software.

Figure 4 and 5 present a comparison between the errors measured from GCPs. GCPs coordinates are determined from

\footnotetext{
${ }^{1}$ GUI tool in Pix4D software to measure GCP coordinate on images
}
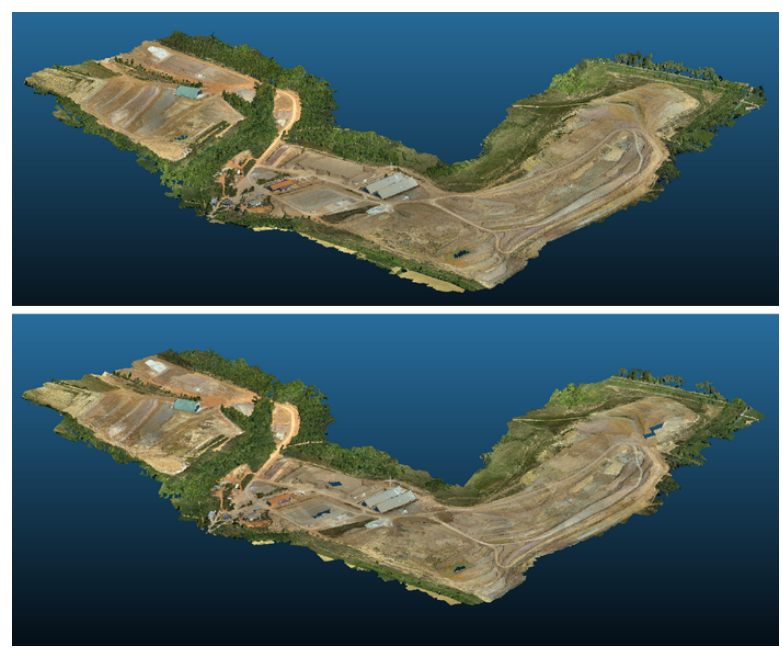

Figure 3. 3D dense point cloud reconstructed by Pix4D (above) anf FORMap (below) from the UX11 dataset.

FORMap and Pix4D processing results. The error is the Euclidean distance between photogrammetry determined GCPs and measured GCPs. We observe that results given by the two software don't have any significant systematic error, except a small bias in $Z$ for Pix4D in the senseFly dataset (see Figure 5, bottom).

Comparison of the GCPs error statistics between two software is shown in Table 3. We observe slightly more accurate results given by Pix4D in planar coordinates for the UX11 dataset $([8,7] \mathrm{cm}$ vs $[11,10] \mathrm{cm})$ and for the senseFly dataset $([8,7] \mathrm{cm}$ vs $[5,5] \mathrm{cm})$. The vertical error component given by FORMap is slightly higher for the UX11 dataset $(11 \mathrm{~cm}$ vs $9 \mathrm{~cm})$, but are comparable in the senseFly dataset (both software reach $6 \mathrm{~cm}$ ).

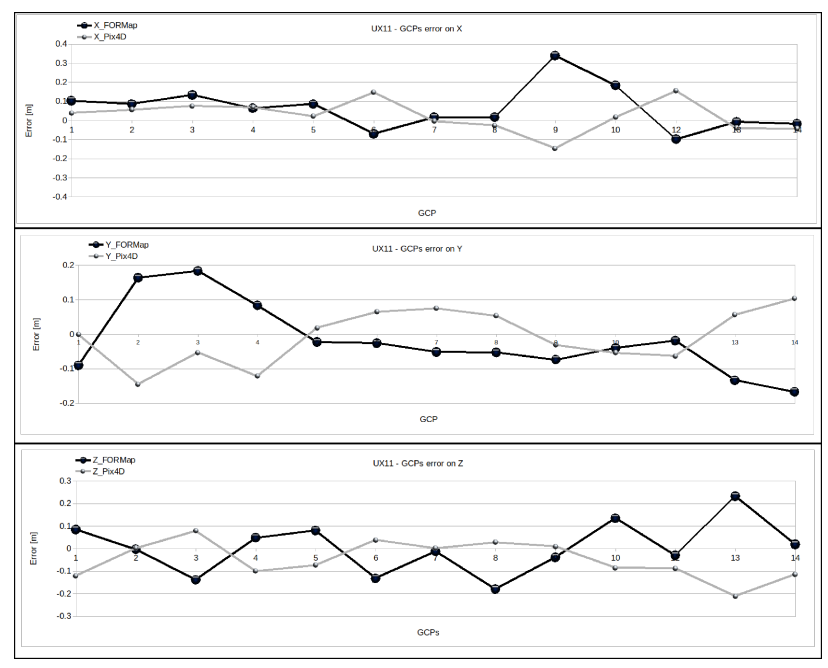

Figure 4. Accuracy assessment on GCPs of UX11 dataset. From the top : Euclidean distance, in X, in Y, in Z. Black line :

FORMap, Grey line : Pix4D. The horizontal axis indexes the GCP number and the vertical axis is centered at 0 in all figures.

However, FORMap returns a final result much rapidly than Pix4D without much trade off on the accuracy. The comparison of whole processing time between FORMap and Pix4D for the UX11 dataset (Figure 6) reveals that FORMap is significantly faster. While Pix4D takes 4.5 hours to process 2159 images of $20 \mathrm{Mpx}$ at $1 / 8$ re-sampling rate (to get $3 \mathrm{D}$ dense point cloud 


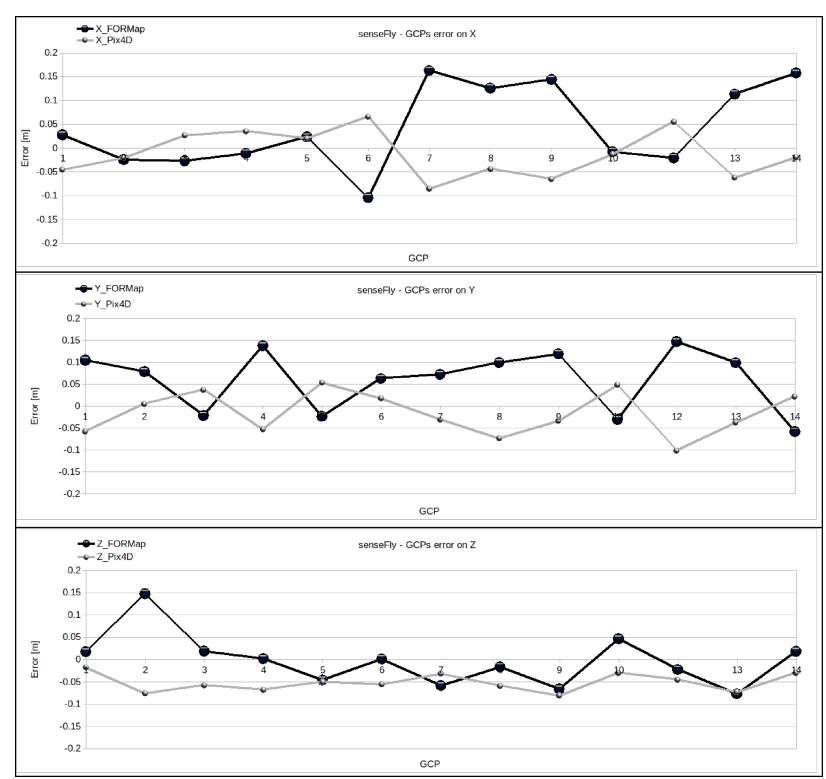

Figure 5. Accuracy assessment on GCPs of senseFly dataset. From the top : Euclidean distance, in X, in Y, in Z. Black line : FORMap, Grey line : Pix4D. The horizontal axis indexes the GCP number and the vertical axis is centered at 0 .

and orthophoto) with its Rapid configuration, FORMap takes only 1.2 hours to deliver results at the same resolution. As we mentioned above, this is an interesting feature for specific applications that require a very low processing time to obtain a acceptable result than a very high accuracy.

\begin{tabular}{|c|c|lll|l|l|}
\cline { 3 - 6 } \multicolumn{2}{c|}{} & \multicolumn{2}{c|}{ UX11 } & \multicolumn{2}{c|}{ senseFly } \\
\cline { 3 - 7 } \multicolumn{2}{c|}{ RMS } & $\mathrm{X}$ & 0.11 & 0.08 & 0.08 & 0.05 \\
\cline { 2 - 6 } Error & Y & 0.10 & 0.07 & 0.07 & 0.05 \\
\cline { 2 - 6 } & $\mathrm{Z}$ & 0.11 & 0.09 & 0.06 & 0.06 \\
\hline \multirow{3}{*}{ Mean } & $\mathrm{X}$ & 0.06 & 0.03 & 0.04 & -0.01 \\
& $\mathrm{Y}$ & -0.02 & -0.008 & 0.06 & -0.02 \\
\cline { 2 - 6 } & $\mathrm{Z}$ & 0.005 & -0.05 & -0.003 & -0.05 \\
\hline \multirow{3}{*}{ Sigma } & $\mathrm{X}$ & 0.11 & 0.08 & 0.08 & 0.05 \\
\cline { 2 - 6 } & $\mathrm{Y}$ & 0.10 & 0.07 & 0.07 & 0.05 \\
\cline { 2 - 6 } & $\mathrm{Z}$ & 0.11 & 0.08 & 0.06 & 0.02 \\
\hline
\end{tabular}

Table 3. Statistic on GCPs error processing by Pix4D and FORMap on the UX11 and senseFly dataset. Measurement unit is $m$.

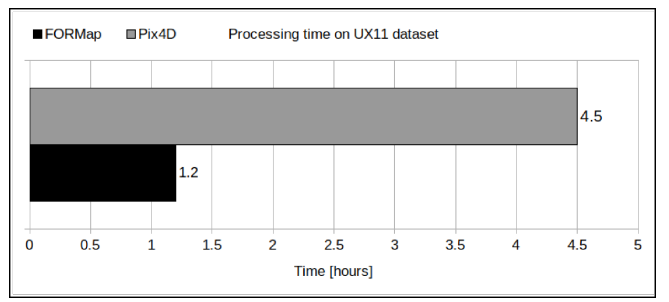

Figure 6. Comparison on processing time of UX11 dataset. Black line : FORMap, Grey line : Pix4D

\subsection{The Inieres and the Highway dataset}

The Inieres and Highway datasets share a same scene geometry: a corridor mapping. Inieres dataset is a concatenation of some corridor lines in different flight directions, which reach a total length of $7 \mathrm{~km}$. Data is acquired by Delair-Tech UX11 fixed wing UAV with its integrated camera. Image positioning is post processed. Attitude data is directly given by an IMU in flight controller.

A Microdrones md4-1000DG ${ }^{2}$ quadcopter was used to acquired the Highway dataset. Dataset contains 357 images in two axes, with $85 \%$ overlap and $85 \%$ sidelap. Direct georeferencing data is given by the integrated Applanix APX-15 module. To test the sensitivity of FORMap with errors in image position and orientation, we did not post process the navigation data of the Highway dataset.

Figure 7 and 8 show the results of FORMap for the Ineres and Highway dataset, respectively. FORMap takes 1.4 hours to process the Ineres dataset and 15 minutes for the Highway dataset. It yields to a processing time of around 2.5 seconds per images. The image re-sampling rate was $1 / 5$ for the Highway dataset and $1 / 3$ for the Ineres dataset (original image resolution is mentioned in the Table 1 and 2).

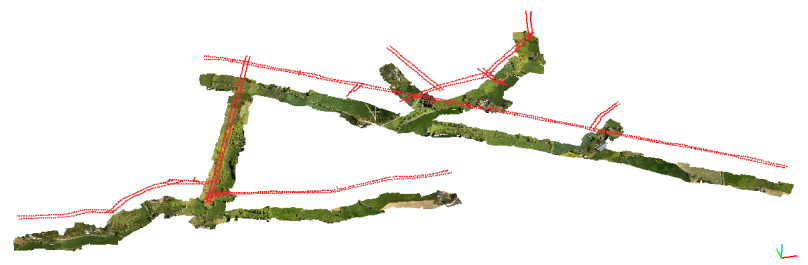

Figure 7. The 3D dense point cloud reconstructed by FORMap from 1976 images from the Ineres dataset. Flight path is presented in red dots.
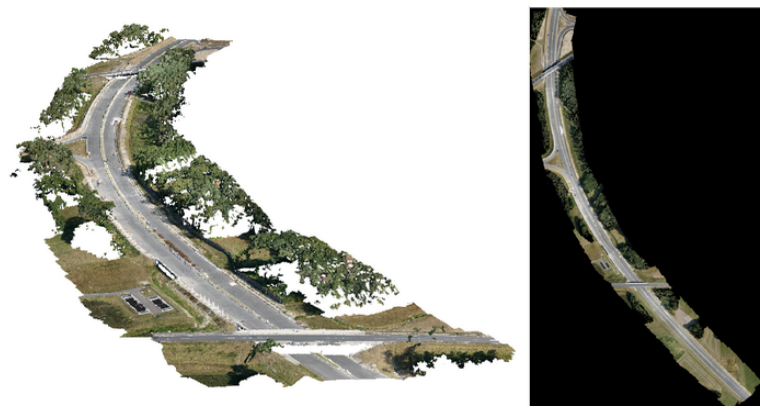

Figure 8. Reconstruction results of FORMap for the Highway dataset. Left: 3D dense point cloud, right: Orthophoto.

\section{ROBUSTNESS ANALYSIS}

In this section, we present two types of test that were conducted to evaluate the robustness of FORMap with respect to:

- Degradation of position information associated to each image;

- High grazing angles of the camera.

The first series of test was done by simulating realistic GNSS errors on the Highway dataset (section 3.3), and by observing the consistency of the reconstructed tie-point point cloud.

\footnotetext{
${ }^{2}$ https://www.microdrones.com/fr/systemesintegres/mdmapper/mdmapper1000dg/
} 
From a post processed PPK (Post Processed Kinematic) position and raw GNSS data of the Highway dataset, the uncertainty of image positions is simulated at different levels. In modeling the difference between these two GNSS data by an ARMA (autoregressive-moving-average) process, we were able to produce several uncertainty levels in playing with the noise gain. Figure9 presents three simulated GNSS positions with different uncertainty levels.

We used FORMap with these simulated GNSS data in input in order to test the the robustness with respect to position uncertainty. One can check in Figure 10 that FORMap succeeded to reconstructed the scene despite of high uncertainty in input image positions. However, the absolute geo-referencing accuracy still depends on the accuracy of the input data. Figure 11 shows the position error with respect to the PPK reference after BBA.

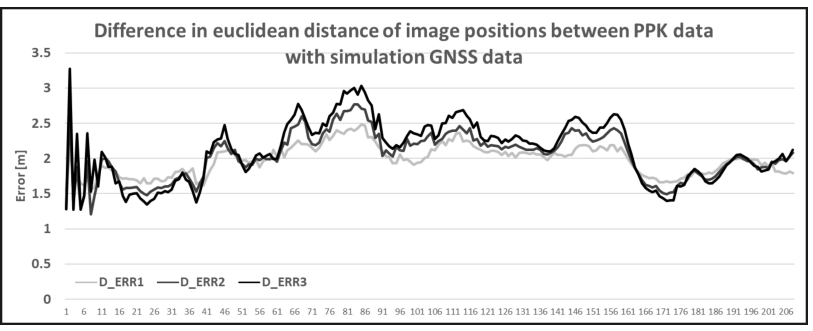

Figure 9. The difference in euclidean distance of image positions between post processed and simulated GNSS data.
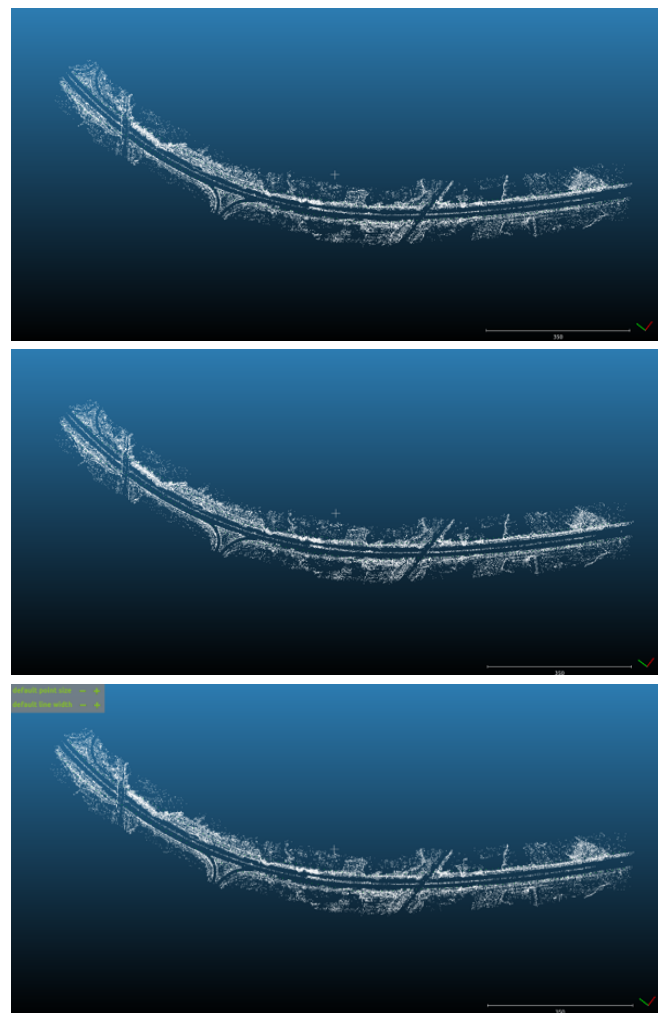

Figure 10. Tie-point cloud after BBA in the case of position uncertainty level 1 (top), level 2 (middle) and 3 (bottom). One can check that the structure of the tie-point is stable even with a level 3 of disturbance acting on the position.

The second test was done using a Microdrones md4-1000DG with an oblique camera angle (mounted at $45^{\circ}$ respect to the local planar). Figure 12 shows some images in the Oblique

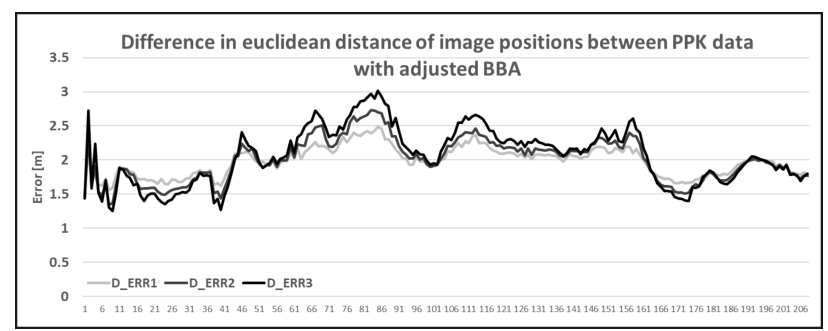

Figure 11. The difference in euclidean distance of image positions between post processed PPK GNSS and after adjusted BBA.

dataset. We could observe that images contain many repetitive pattern that could cause a challenge in tie-point extraction.

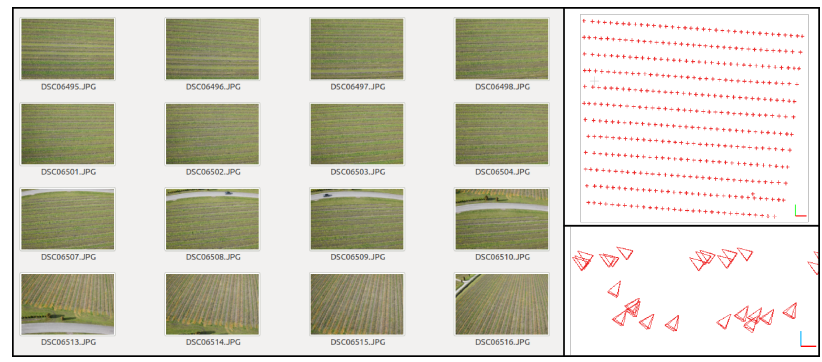

Figure 12. The Oblique dataset. Images acquired with a $45^{\circ}$ oblique angle mounted camera.

We tried to process the Oblique dataset with Pix4D and FORMap. Non post processed GNSS and attitude data is given as input. Pix4D couldn't calibrated all images in the dataset despite of the highest image resolution used in tie-points extraction step as show on 13. Note that a series of downsampling image resolution for tie-point extractions process in Pix4D were also tested. FORMap however succeeded to adjust all images EO parameters and to reconstruct the scene (Figure 14) at a $1 / 5$ th image re-sampling rate.

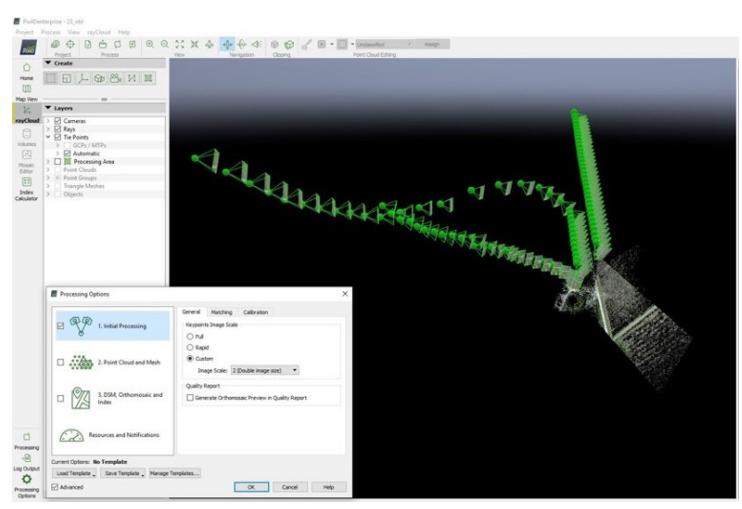

Figure 13. Pix4D could not calibrate all the images of the Oblique dataset despite of highest resolution setting in tie-point extraction.

\section{CONCLUSION AND PERSPECTIVES}

\subsection{Conclusions}

In this article, FORMap - a photogrammetry solution aiming at rapid mapping from UAV data including DG was presented. 

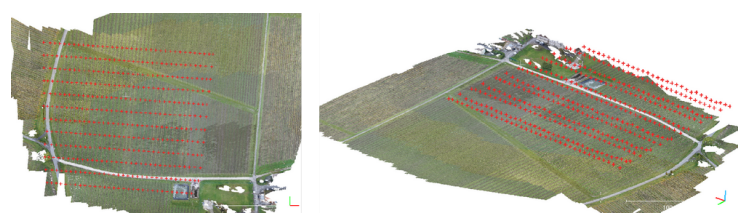

Figure 14. FORMap success to calibrate all images in the Oblique dataset despite of non post processed GNSS data.

FORMap makes use of approximate EO and IO parameters to accelerate the processing, with a light trade off in geometric accuracy. It is therefore suitable for applications which request a simple, highly automatic and rapid 3D scene and orthophoto restitution.

The performance and robustness of FORMap were assessed in four different scenario: two classical grid flight on the same scene with different UAV system and two corridor mapping scenes. The result accuracy and the processing time was investigated in the UX11 dataset with GCPs and compared with Pix4D. Results show that FORMap is far more better in processing time with a very light degradation in the GCP accuracy, in compararison with Pix4D. However, statistical analysis on the GCPs accuracy don't show any clear systematic error in both FORMap and Pix4D results.

\subsection{Perspectives}

FORMap employs a BBA using initial approximated EO and IO parameters associated to each image to accelerate the process. These parameters can be easily obtained, even on a small UAV system. We shown FORMap robustness and performance through several tests. Some amelioration tracks could be considered in the near future to improve the reconstruction and georeferencing accuracy such as :

\section{The camera distortion calibration :}

Current version of FORMap support two camera distorsion calibration model : a three parameters radial and five parameters ( 3 factors radials +2 factors tangential). Some hard configuration such as corridor presented in section 3.3 could lead to some systematic error such as a calibration bowl effect (James, Robson, 2014, Zhou et al., 2019). A high degree distorsion model combine with constraint on GNSS observations could be considered to eliminate this effect (Tournadrea et al., 2015).

2. The lever-arm GNSS-camera estimation :

The level-arm between GNSS antenna phase center and camera optical center is usually calibrated by the UAV constructor, and are taken in account when geotagging image. However, this offset value could be varied by mechanical impact when operating the UAV such as vibration, crash... An estimation of this offset during the BBA process could be considered for better geo-referencing of the scene (Daakir, 2017).

3. The improvement of tie points extraction :

The post processing of the FORMap result for extracting a new set of tie-points that are optimized for photogrammetric processing could be considered for high-precision applications (Truong Giang et al., 2018, Nguyen et al., 2017).
4. The improvement of 3D dense point cloud :

Some post processing such as denoising ${ }^{3}$ could be applied to the final 3D dense point cloud to enhance the precision on unregulated surface.

\section{REFERENCES}

Association, C. . I. P. et al., 2010. Exchangeable image file format for digital still cameras: Exif Version 2.3. CIPA DC-008 Translation-2010.

Ball, A., Darlington, M., 2007. Briefing Paper: The Adobe eXtensible Metadata Platform (XMP). UKOLN research organization.

Bianco, S., Ciocca, G., Marelli, D., 2018. Evaluating the performance of structure from motion pipelines. Journal of Imaging, 4(8), 98.

Daakir, M., 2017. Centimetric absolute localization using Unmanned Aerial Vehicles with airborne photogrammetry and onboard GPS. PhD thesis.

Eisenbeiß, H., 2009. UAV photogrammetry. PhD thesis, ETH Zurich.

Gabrlik, P., Jelinek, A., Janata, P., 2016. Precise multi-sensor georeferencing system for micro UAVs. IFAC-PapersOnLine, 49(25), 170-175.

James, M. R., Robson, S., 2014. Mitigating systematic error in topographic models derived from UAV and ground-based image networks. Earth Surface Processes and Landforms, 39(10), $1413-1420$.

Mian, O., Lutes, J., Lipa, G., Hutton, J. J., Gavelle, E., Borghini, S., 2015. Direct Georeferencing on small unmanned aerial platforms for improved reliability and accuracy of mapping without the need for ground control points. ISPRS - International Archives of the Photogrammetry, Remote Sensing and Spatial Information Sciences, XL-1/W4, 397-402. https://www.int-arch-photogrammremote-sens-spatial-inf-sci.net/XL-1-W4/397/2015/.

Nguyen, T., Pierrot-Deseilligny, M., Muller, J.-M., Thom, C., 2017. Second Iteration of Photogrammetric Pipeline to Enhance The Accuracy of Image Pose Estimation. The International Archives of Photogrammetry, Remote Sensing and Spatial Information Sciences, 42, 225.

Peppa, M., Hall, J., Goodyear, J., Mills, J., 2019. Photogrammetric assessment and comparison of DJI Phantom 4 pro and phantom 4 RTK small unmanned aircraft systems. ISPRS Geospatial Week 2019.

Pérez, M., Agüera, F., Carvajal, F., 2013. Low cost surveying using an unmanned aerial vehicle. Int. Arch. Photogramm. Remote Sens. Spat. Inf. Sci, 40, 311-315.

Rabah, M., Basiouny, M., Ghanem, E., Elhadary, A., 2018. Using RTK and VRS in direct geo-referencing of the UAV imagery. NRIAG Journal of Astronomy and Geophysics, 7(2), $220-226$.

\footnotetext{
${ }^{3}$ https://www.geown.io/denoising/
} 
Rupnik, E., Daakir, M., Deseilligny, M. P., 2017. MicMac-a free, open-source solution for photogrammetry. Open Geospatial Data, Software and Standards, 2(1), 14.

Sauerbier, M., Siegrist, E., Eisenbeiss, H., Demir, N., 2011. The practical application of uav-based photogrammetry under economic aspects. Proceedings of the International Conference on Unmanned Aerial Vehicle in Geomatics (UAV-g), 38, Copernicus, $45-50$.

Specification, X., 2005. Adobe systems inc.

Taddia, Y., Stecchi, F., Pellegrinelli, A., 2019. Using DJI Phantom 4 RTK drone for topographic mapping of coastal areas. Int. Arch. Photogramm. Remote Sens. Spat. Inf. Sci, 625630 .

Toldo, R., Gherardi, R., Farenzena, M., Fusiello, A., 2015. Hierarchical structure-and-motion recovery from uncalibrated images. Computer Vision and Image Understanding, 140, 127 143.

Tournadrea, V., Pierrot-Deseilligny, M., Faure, P., 2015. UAV linear photogrammetry. International Archives of the Photogrammetry, Remote Sensing \& Spatial Information Sciences, 40.

Truong Giang, N., Muller, J.-M., Rupnik, E., Thom, C., PierrotDeseilligny, M., 2018. Second Iteration of Photogrammetric Processing to Refine Image Orientation with Improved TiePoints. Sensors, 18(7), 2150.

Zhou, Y., Rupnik, E., Faure, P., Pierrot-Deseilligny, M., 2018. Gnss-assisted accurate corridor mapping with small uav.

Zhou, Y., Rupnik, E., Meynard, C., Thom, C., PierrotDeseilligny, M., 2019. Simulation and analysis of photogrammetric UAV image blocks: influence of camera calibration error. ISPRS Annals of Photogrammetry, Remote Sensing \& Spatial Information Sciences, 4. 